\title{
Adecuación de la Planeación Didáctica como Herramienta Docente en un Modelo Universitario Orientado al Aprendizaje
}

\author{
Educational Planning Adequacy as Undergraduate Teaching \\ Tool in a Learning-oriented Education Model
}

\author{
Claudia Ascencio Peralta * \\ Universidad Latinoamericana
}

\begin{abstract}
La planeación didáctica implica la organización de un conjunto de ideas y actividades que permiten desarrollar un proceso educativo con sentido, significado y continuidad. Constituye un modelo o patrón que permite enfrentar de forma ordenada y congruente, situaciones a las que el estudiante se enfrentará en su vida profesional o cotidiana y en el caso del profesor, a su práctica docente.

La Universidad Latinoamericana (ULA) no contaba con un formato estandarizado de planeación didáctica, por lo que se hizo un análisis de los diferentes formatos empleados por los docentes de todas las licenciaturas presenciales de uno de sus Campi en distintos ciclos escolares, hasta obtener una propuesta que fusionó componentes de los syllabi y los planes de clase en un formato vanguardista y adecuado al modelo educativo de esta institución. El análisis de los formatos y las cartas descriptivas de algunos docentes permitieron determinar los ajustes requeridos al formato, del responsable del llenado de algunos apartados, así como hacer sugerencias de recursos adicionales requeridos por los docentes para facilitar una docencia de calidad.
\end{abstract}

Descriptores: Planeación didáctica, Modelo educativo ULA, Metas de aprendizaje, Evaluación de aprendizajes, Calidad educativa.

Educational planning involves the organization of a set of ideas and activities that develop an educational process with meaning, significance and continuity. It is a model or pattern that can deal in an orderly and consistent way, with situations that students will face in their professional or everyday life and in the case of the teacher, with his or her teaching practice.

The Universidad Latinoamericana (ULA) did not have a standardized educational planning format, so that formats used by teachers in different school years were analyzed, until a proposal that combined components of syllabi and lesson plans in a modern and appropriate format were produced, one adapted to the educational model of this institution. The analysis of the formats and educational planning from some teachers allowed to determine the required adjustments to the format, the responsible for filling some sections as well as to make suggestions for additional resources required by teachers to provide quality teaching.

Keywords: Educational planning, ULA's educational model, Learning goals, Learning assessment, Quality of education.

\footnotetext{
*Contacto: claudia.ascencio@ula.edu.mx
}

ISSN: 1696-4713

www.rinace.net/reice/

revistas.uam.es/reice
Recibido: $\quad 15$ de septiembre 2015

$1^{\text {a }}$ Evaluación: 13 de noviembre 2015

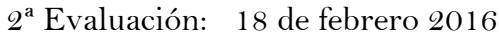

Aceptado: 1 de abril 2016 


\section{Introducción}

Aumentar la calidad y la equidad educativa ha sido considerado un elemento clave para lograr la transformación de México, lo cual está reglamentado en el Programa Sectorial de Educación 2013-2018 del actual gobierno y alineado a los estándares de calidad recomendados por la UNESCO (2005) y la OIE. En esta tarea, los profesores ocupan un papel fundamental. Como en muchos países latinoamericanos y europeos, el énfasis de la docencia se ha enfocado en el aprendizaje efectivo de los estudiantes a partir de un cuerpo docente que lo asegure y un sistema educativo que lo sustente. Para realizar su tarea docente, los profesores necesitan contar con los mejores apoyos que orienten su labor de enseñanza, especialmente a nivel de la educación superior, debido a que los docentes no suelen contar con formación pedagógica previa. Las principales herramientas de organización de la docencia empleadas en México a nivel superior incluyen a la planeación didáctica o carta descriptiva, el syllabo y el plan de clase, documentos complementarios a los programas académicos y el plan de estudios.

La institución educativa (ULA) tiene como misión formar individuos exitosos mediante programas académicos de alta calidad, que incluyen práctica profesional, el empleo de tecnologías educativas de vanguardia y el desarrollo conocimientos científicos y tecnológicos, así como actitudes que fortalecen en el egresado su quehacer profesional. Prepara tanto a jóvenes recién egresados de la preparatoria como a personas adultas que trabajan. Su modelo educativo "Educar para transformar: conocimiento útil" se orienta al aprendizaje de los estudiantes, conformando un modelo ecléctico, en el que el docente tiene un papel central en el diseño del plan maestro que guiará las actividades académicas de enseñanza-aprendizaje, que se plasman en la planeación didáctica de la asignatura.

Dicho modelo está orientado a promover en el estudiante una formación integral, que involucra que adquiera los saberes que demanda el campo laboral, así como habilidades clave para la vida que le permitan desenvolverse como un aprendiz autónomo; además integra diversos aspectos relacionados con el proceso de enseñanza-aprendizaje, tales como el papel del estudiante y del docente en el proceso educativo, los recursos educativos y tecnológicos e incorpora aportaciones teóricas y metodológicas derivadas de los avances de las ciencias cognitivas, de la psicología educacional y de la pedagogía, entre otras ciencias.

Como lo indica su filosofía institucional, la ULA se encuentra inmersa en la búsqueda de la calidad educativa de sus estudiantes, por lo que revisa de manera habitual sus procesos internos, que ayudan a detectar puntos de mejora. Esto incluye los aspectos relacionados con la práctica docente dentro y fuera del aula, así como las necesidades de capacitación de su personal. En este sentido, Casanova (2012) define el concepto de calidad educativa como ... algo casi intangible, pero variable y dinámico, que cambia con el tiempo y las personas que lo conceptualizan, con sus ideologías, creencias y supuestos pedagógicos vigentes... La calidad total es la mejora progresiva en la forma de hacer las cosas, aun cuando hayan sido realizadas sin errores, lo que se enfoca en la mejora permanente y esto es especialmente válido al hablar de educación.

Preocupados por contar con un formato de planeación didáctica de vanguardia, que permitiera aportar la información útil para el estudiante y favorecer la economía de 
trabajo de los docentes, nos dimos a la tarea de realizar un análisis de las diferentes modalidades de carta descriptiva/planeación didáctica empleadas en distintos periodos escolares en uno de sus Campi, así como comparar este diseño con otras estrategias empleadas por otras universidades, incluyendo el uso del syllabo y el plan de clase; para proponer un formato que mejorara tanto sus componentes de forma, fondo, secuencia y congruencia con el modelo de la institución educativa.

\section{Marco teórico}

Se entiende a la planeación didáctica como la organización de un conjunto de ideas y actividades que permiten desarrollar un proceso educativo con sentido, significado y continuidad. Constituye un modelo o patrón que permite al docente enfrentar su práctica de forma ordenada y congruente (SEP, 2009). Su elaboración implica analizar y organizar los contenidos educativos, determinar los objetivos, intenciones y propósitos educativos a lograr; además de establecer la secuencia de actividades en el tiempo y espacio.

En forma conjunta, la información que contienen conforma un plan de acción integral, que permite dar claridad a los fundamentos educativos que orientarán el proceso de enseñanza aprendizaje. Generalmente están conformados por un formato preestablecido que permite la homogenización de documentos entre docentes y entre escuelas y facultades de la misma institución educativa.

La manera en la que el docente estructura la enseñanza, la distribución del tiempo en el aula, las actividades que asigna a los estudiantes para trabajo en el aula, los recursos educativos, las estrategias y mecanismos de evaluación y sus propias expectativas de la clase son algunas de las perspectivas que hoy en día se consideran como parte de una enseñanza eficaz (Murillo, Martínez-Garrido y Hernández-Castilla, 2011). A esto se suma el andamiaje necesario para que el alumno construya los conocimientos por apropiación de los contenidos, la práctica para aplicarlos y emplearlos en diversas situaciones, así como el papel del docente como maestro artesano, que transforma al aprendiz en un experto, lo que acentúa la importancia de las características, conocimientos y valores que debe tener el maestro en su labor docente y que debe ser reflejado dentro y fuera del aula mediante la planeación de su asignatura.

La planeación no se debe llevar a cabo sólo como una actividad técnica limitada a llenar formatos, sino que debe traducirse en intenciones educativas que son el resultado del análisis de varios componentes, entre los que destacan elementos contextuales como la cultura y las condiciones socioeconómicas del entorno donde los estudiantes desempeñan las habilidades cognitivas y aprendizajes previos que han podido desarrollar en etapas escolares anteriores o en su desarrollo (SEP, 2013). La elaboración y el llenado de este instrumento deben ser considerados como un aspecto primordial del aseguramiento de la calidad académica por parte de las instituciones educativas, por lo que es de suma importancia que el instrumento cuente con todos los requisitos necesarios para darle validez y que los usuarios del mismo lo sepan llenar y aplicar adecuadamente.

Casanova (2012) aboga por un currículum que incluya lo importante para disponer de una formación básica integral, que favorezca la transferencia de aprendizajes al contexto de la vida real, que le permitan al estudiante perfilarse como especialista en ser persona (como lo demanda la vida) y especialista en ser profesional (como lo demanda la 
sociedad). Por ello, enfatiza el empleo de metodologías educativas que permitan aproximar el aprendizaje a cada sujeto, de acuerdo a sus características individuales, mediante el uso de múltiples recursos didácticos. El maestro es quien debe descubrir cuál es la metodología más adecuada para cada persona a la que está educando, así como el sistema de evaluación que verdaderamente refleje su desempeño a lo largo de la asignatura y no sólo los conocimientos que escribe una vez al mes en un examen.

Entre las ventajas que se derivan de la elaboración y uso de la planeación didáctica de un curso están el facilitar a los maestros la realización de sus funciones docentes, sobre todo en el caso de profesores inexpertos; establecen condiciones para que los alumnos adquieran una formación homogénea; se tiene un punto de referencia para seleccionar y entrenar al personal docente, así como para escoger y emplear recursos didácticos y se orienta desde el inicio del curso, a los alumnos que participan con él (Haro del Real, 1994).

En realidad, no hay una fórmula mágica que defina cuál es la metodología más eficaz para favorecer el aprendizaje y por ello se recomienda una combinación de muchas, con base en la creatividad del docente (Murillo, Martínez-Garrido y Hernández-Castilla, 2011), pero que respondan a conseguir un propósito educativo claro.

En México, algunas universidades emplean el syllabus, cuyo propósito también es definir los contenidos y logros o resultados de aprendizaje e informan a los estudiantes sobre la forma en que el docente o instructor organizó su curso o asignatura. Además sirve para transmitir las expectativas del docente, mostrar una imagen panorámica del curso, establecer un contrato con los estudiantes respecto a las políticas, reglas, requerimientos y procedimientos del curso; ayudar a los estudiantes a evaluar que tan preparados están para tomarlo, identificando áreas de conocimiento que son prerrequisitos para el curso, orientar a los estudiantes respecto a los recursos requeridos, ofrecerles tiempos de tutorías y para comunicar sus metas y contenidos a sus colegas. Suelen ser formatos menos estructurados y por lo tanto no son homogéneos.

Por otro lado, el plan de clase es el instrumento más específico de la planeación de un curso, que integra las actividades que deben realizarse antes de la sesión, al inicio, durante el desarrollo y en el cierre de la misma, por lo que incluye de manera detallada las actividades a realizar y los recursos que se emplean en la sesión. Este documento debe elaborarse para cada una de las sesiones que conforman un curso o asignatura, lo que lo torna complicado, debido a que en un semestre puede requerir más de 50 planes de clase, si una asignatura se imparte 3 veces por semana a lo largo de 16 o 18 semanas académicas.

\section{Justificación}

Al inicio de cada ciclo escolar, se les pide a los docentes de la ULA entregar la planeación didáctica de la(s) asignatura(s) que impartirán. Este documento se revisa por parte de los coordinadores o directores del programa académico, en su caso se autoriza, se firma y se resguarda copia del documento en la coordinación académica. Este documento queda a disposición de las autoridades educativas con fines de evaluación del desempeño docente.

La planeación didáctica también se entrega a los estudiantes en un formato electrónico de modo que puedan ocuparlos como guía del avance programático de cada asignatura. 
Sin embargo, el formato suele ser entregado por los docentes con errores de diferente tipo y magnitud, incluyendo datos incorrectos en la ficha de identificación, en la redacción de objetivos, en la distribución de temas y subtemas de acuerdo a la calendarización de la asignatura, modificación de la bibliografía básica, poca variedad en actividades frente a grupo y en el manejo de una escasa cantidad de recursos, por lo que la utilidad que ha tenido el formato ha sido muy limitada en los siguientes aspectos:

- Para los docentes y estudiantes como una guía de orientación de la asignatura.

- Para las coordinaciones académicas como mecanismo de evaluación del desempeño docente.

Además, el machote del formato sufrió variaciones de contenido y forma a lo largo de diferentes periodos escolares por indicaciones y ajustes sugeridos por diferentes áreas directivas, pero sin la capacitación respectiva, lo que propició que los docentes desconocieran qué información debían incluir en los diferentes apartados. Finalmente estos problemas se convirtieron en una sensación de malestar, disgusto o frustración por parte de los docentes, quienes acabaron entregando el formato como un mero trámite administrativo y no como su guía de orquestación de clase.

El propósito del presente trabajo fue analizar las variantes de información y la forma de llenado de los formatos de planeación didáctica utilizados a lo largo de 2 años en la institución educativa, para:

- Definir cuáles rubros de información son indispensables en la planeación didáctica como guía de clase para los docentes y estudiantes.

- Detectar los principales errores de captura de información o en empleo de estrategias didácticas y recursos, cometidos por los docentes de las diferentes escuelas de uno de los Campi.

- Hacer una propuesta de formato institucional mejorada, acorde a su modelo educativo y a las habilidades de gestión de su planta docente.

\section{Materiales y métodos}

En función de los objetivos planteados, se optó por la realización de un estudio descriptivo de corte cualitativo, a partir de la revisión y el análisis de 20 ejemplos de formatos de planeación didáctica empleados en los cuatro últimos ciclos escolares (20141 a 2015-2), que fueron entregados a las coordinaciones académicas al inicio de cada ciclo escolar, por docentes de las diferentes licenciaturas presenciales del campus Cuernavaca (Médico Cirujano, Cirujano Dentista, Psicología, Nutrición). Se comparó la información con el programa académico de cada licenciatura, para evaluar la consistencia de la información respecto a la transcripción de los datos de identificación de la asignatura, objetivo general, los temas y subtemas, estrategias de evaluación y bibliografía básica.

Además se analizaron los componentes que cada docente añade a la planeación didáctica, como son los objetivos específicos por tema, la distribución de temas y subtemas de acuerdo al número total de horas a impartir por ciclo escolar, los recursos propuestos, las actividades de aprendizaje, la forma de evaluación y las bibliografías complementarias. 
Se detectaron los principales errores de llenado de información, encontrando algunas ventanas de oportunidad para mejorar tanto aspectos de llenado (transcripción de información), como de estructura del formato, que se comentarán en las siguientes secciones.

Así mismo, se hizo una revisión por internet de formatos de planeación didáctica o cartas descriptivas a nivel de universidades y se revisó el diseño y la estructura de los syllabi empleados por algunos centros educativos en México, además de retomar los componentes del plan de clase, para finalmente definir un modelo de planeación didáctica adecuado a las necesidades de la institución educativa, que fusiona componentes de los tres instrumentos de acuerdo a su modelo educativo.

\section{Resultados}

\subsection{Elementos fundamentales de la planeación didáctica institucional}

Las secciones contenidas de manera permanente en los formatos institucionales de planeación didáctica revisados incluyeron:

$\checkmark$ Ficha de identificación de la asignatura: nombre del campus, ciclo escolar, nombre y clave de la asignatura, horas de trabajo frente a docente e independientes, créditos, objetivo general de la asignatura, nombre y firma del docente y visto bueno del director o coordinador del programa académico.

$\checkmark$ Contenidos con temas y subtemas, número de sesión y fecha calendario de impartición.

$\checkmark$ Sección de objetivos específicos del tema.

$\checkmark$ Sección de criterios de evaluación.

$\checkmark$ Bibliografía.

$\checkmark$ Ficha de identificación de la asignatura o curso

De acuerdo a Zabalza (2004), las planeaciones didácticas deben comenzar con datos descriptivos de la asignatura: nombre, clave, tipo de asignatura, ciclo escolar, número de créditos, horas de impartición frente a docente $\mathrm{y}$ de trabajo independiente, prerrequisitos, nombre del docente, horas de tutoría y lugar. También recomienda incluir el sentido de la asignatura en el perfil de la titulación, en el que se explica la importancia formativa de la materia en el Plan de Estudios en el que está ubicada. Esto ayuda a los estudiantes a entender lo que cada asignatura les aporta en su proceso de formación como futuros profesionales.

Las planeaciones didácticas de la institución educativa fueron modificándose en cuanto a la inclusión de algunos apartados, como el carácter de la asignatura; no se incluyó en ningún formato la seriación y en las dos últimas versiones se incluyó el perfil docente y la contribución de la asignatura al perfil de egreso.

Algunos de los errores más comúnmente cometidos por los docentes en el llenado de esta ficha de identificación, fueron el nombre del campus (Cuernavaca, Morelos, Ciencias de la Salud), el llenado de horas frente a docente e independientes por semana o por semestre, según se encuentren registradas en el plan de estudios; el definir el carácter de 
la asignatura como básica, de integración, clínica, etc., en lugar de obligatoria u optativa y el desconocer los créditos y la clave de la asignatura.

Por ello, la primera sugerencia a la institución educativa fue que el llenado de este apartado recaiga sobre el dueño del programa académico; es decir, el director o coordinador del programa. Por lo tanto, se dividió y separó la secuencia de presentación de la información de la ficha de identificación en un apartado de información no modificable o "plantilla estandarizada", del resto de la información. Esta plantilla debe ser llenada por el coordinador académico (diferenciada con color gris). En este apartado se integró el temario y la bibliografía básica de la asignatura, ya que también son componentes no modificables y que deben mantener total correspondencia entre el plan de estudios, el programa académico y la planeación didáctica (figura 1).

FOMATO DE PLANEACIÓN DIDÁCTICA

Programas Presenciales. Dirección General de Operaciones

1 DATOS DE IDENTIFICACIÓN DE LA ASIGNATURA (Para ser llenado por el coordinador del programa)

\begin{tabular}{|c|c|c|c|c|c|c|}
\hline Campus: & \multicolumn{4}{|c|}{ Nivel educativo: } & \multicolumn{2}{|c|}{ Programa de estudio: } \\
\hline & \multicolumn{4}{|c|}{ Preparatoria ( ) Licenciatura ( ) Posgrado ( ) } & & \\
\hline \multicolumn{2}{|c|}{ Nombre de la asignatura: } & Clave: & Semestre: & \multicolumn{2}{|c|}{ Prerrequisito: } & Seriada con: \\
\hline \multirow[t]{2}{*}{ Créditos: } & \multicolumn{2}{|c|}{$\begin{array}{l}\text { Horas semanales con } \\
\text { docente: }\end{array}$} & \multirow{2}{*}{\multicolumn{2}{|c|}{$\begin{array}{l}\text { Horas semanales de } \\
\text { aprendizaje independiente: }\end{array}$}} & \multirow{2}{*}{\multicolumn{2}{|c|}{$\begin{array}{l}\text { Total de horas a la } \\
\text { semana: }\end{array}$}} \\
\hline & Teóricas & Prácticas & & & & \\
\hline
\end{tabular}

Perfil docente propuesto en el Plan de Estudios :

Perfil docente propuesto en el Plan de Estudios :

Contribución de la asignatura al perfil de egreso:

Objetivo general de la asignatura:

\section{TEMARIO}

\begin{tabular}{|l|}
\hline Temas y subtemas incluidos en el programa académico: \\
\hline \\
\hline B BIBLIOGRAF́́A BÁSICA
\end{tabular}

3 BIBLIOGRAFÍA BÁSICA

Formato APA:

Figura 1. Modificación del esquema de la ficha de identificación del formato de planeación didáctica

Nota: Con color gris se especifican los rubros que deben ser llenados por los directores o coordinadores académico.

Fuente: Elaboración propia. 
En un apartado distinto en color verde, se incluyeron los componentes modificables de la ficha de identificación como son el ciclo escolar, el nombre y firma del docente y el visto bueno del coordinador y se integraron otros datos como información de contacto del docente, su perfil profesional real, así como los principales escenarios en los que se llevará a cabo el curso (figura 2).

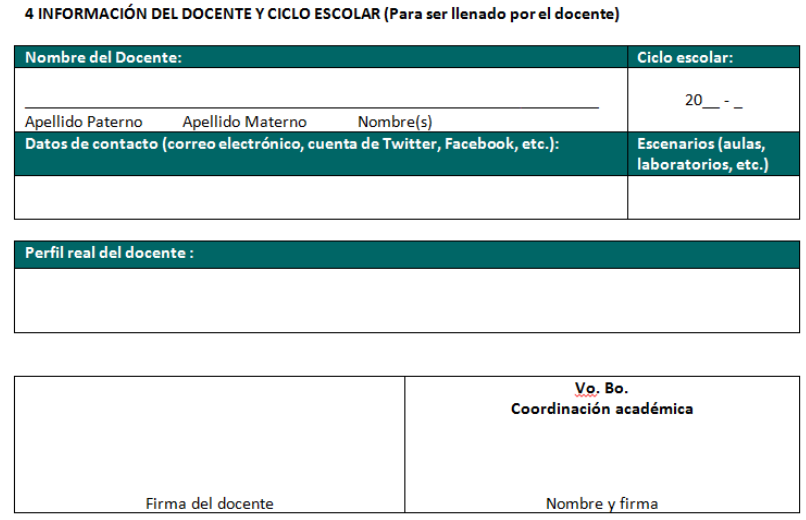

Figura 2. Componentes modificables de la ficha de identificación de la planeación didáctica

Nota: Con color verde, los elementos que deberán ser llenados por el docente.

Fuente: Elaboración propia.

\subsection{Fase de análisis previa a la planeación del curso}

Antes de iniciar el llenado de la planeación didáctica, Fink (2013) recomienda reflexionar sobre el contexto específico de la situación de enseñanza aprendizaje que vivirá el docente, incluyendo aspectos como el número de estudiantes, ubicación de la asigntura en el plan de estudios, número de horas y sesiones a la semana, modalidad, escenarios y el ambiente de clase, en un apartado denominado Factores Situacionales Relacionados con la Asignatura.

En este apartado Fink también incluye las expectativas de aprendizaje del curso en relación con el objetivo general del plan de estudios, de la profesión y de las demandas de la sociedad; la naturaleza de la asignatura (teórica, práctica, convergente o divergente), si hay controversias o cambios recientes de importancia en relación a la temática del curso que se deban integrar, para mantenerla actualizada. Del mismo modo, considera la situación de vida de los estudiantes, sus conocimientos previos, experiencias y sentimientos iniciales que los estudiantes suelen tener respecto a la asignatura, sus metas de aprendizaje, expectativas y estilos preferidos de aprendizaje. Respecto al docente, el reflexionar sobre sus creencias y valores respecto a la enseñanza, su actitud frente a la asignatura, ante los estudiantes, qué tan familiarizado está con la asignatura, cuáles son sus fortalezas y debilidades como profesor.

$\mathrm{El}$ análisis de los factores situacionales relacionados con el grupo previo al llenado del cuerpo principal del formato, fue una importante recomendación que se incluyó en instructivo de llenado dirigido a los docentes, así como en los cursos de capacitación didáctica que reciben los profesores a partir de su ingreso a la institución educativa. 


\subsection{Planeación de la asignatura (cuerpo principal del formato)}

En su artículo sobre realidades y paradigmas de la función docente, Díaz Barriga (2011) describe las características del personal académico de educación superior en México: "Desde el punto de vista pedagógico, el perfil predominante entre los docentes universitarios mexicanos continúa siendo tradicionalista, en tanto que se encuentra centrado en la figura hegemónica del profesor frente al grupo, los métodos de enseñanza siguen siendo prevalentemente expositivos y las estrategias de evaluación generalmente se asocian a la adquisición memorística del conocimiento". "Se observa que un $77 \%$ de los maestros del nivel superior reportan haberse iniciado en el trabajo académico sin experiencia docente previa, y cerca de un $80 \%$ admite contar con una preparación didáctica deficiente, ya que no participa -o lo hace poco- en actividades de formación y actualización para el adecuado ejercicio magisterial”.

En el Campus Cuernavaca de la ULA, 213 docentes imparten clases a nivel licenciatura en carreras afines a la salud. Solo 3 profesores cuentan con estudios de posgrado (maestría) en ciencias de la educación o docencia y otros tres docentes cursaron una licenciatura en pedagogía, lo que representa el $2.8 \%$ del total de profesores que cuentan con experiencia en docencia o educación.

En conclusión, una gran mayoría de los docentes de educación superior en México son profesionistas egresados de licenciaturas, que nunca han realizado estudios específicos que los capaciten para ejercer la docencia. Por ello, la capacitación del profesorado en temas relacionados con la docencia y la gestión escolar, incluyendo la planeación didáctica, es un aspecto de gran importancia para la universidad.

De acuerdo al modelo de Fink, una vez definidos los factores situacionales se deben determinar las metas de aprendizaje; es decir, lo que queremos que los estudiantes se lleven del curso. Con preguntas como: ¿Qué es importante que aprendan y retengan 2 a 3 años después de que terminó el curso o asignatura? ¿Qué clase de habilidades de pensamiento o de aplicación queremos que desarrollen? ¿Cómo podemos ayudarlos a seguir aprendiendo cuando el curso haya terminado?. Incluir no solo actividades de entender y recordar, sino enfatizar el pensamiento crítico, aprender a resolver problemas, cambiar la forma en la que los estudiantes piensan sobre ellos mismos o de otros, y la importancia del aprendizaje autónomo por toda la vida. Sugiere el empleo de la taxonomía para el aprendizaje significativo como marco de referencia (figura 3). Integra seis categorías de aprendizajes que pueden incluirse como metas de aprendizaje importantes de la asignatura o curso (Fink, 2006). Estos aprendizajes son interactivos; es decir, cada clase de aprendizaje puede estimular otros y al revés.

Debido a que el modelo educativo de la institución se centra en los aprendizajes de los estudiantes, la propuesta taxonómica de verbos de Fink se adapta mejor a su modelo, que la redacción de objetivos y metas basadas en la Taxonomía de Bloom, que ha sido la más utilizada por los profesores, especialmente con verbos conceptuales (tabla 1).

La taxonomía propuesta por Fink para el aprendizaje significativo, busca que el docente defina de manera deliberada objetivos de las seis dimensiones propuestas, de modo que los aprendizajes significativos superen a los conceptuales y procedimentales, ya que al planear el curso debe analizar lo que realmente su asignatura puede aportar al perfil de egreso del estudiante, así como a su formación integral como profesionista y ser humano. 
De este modo, a través de todas las asignaturas del plan de estudios, se podría asegurar una formación integral del egresado.

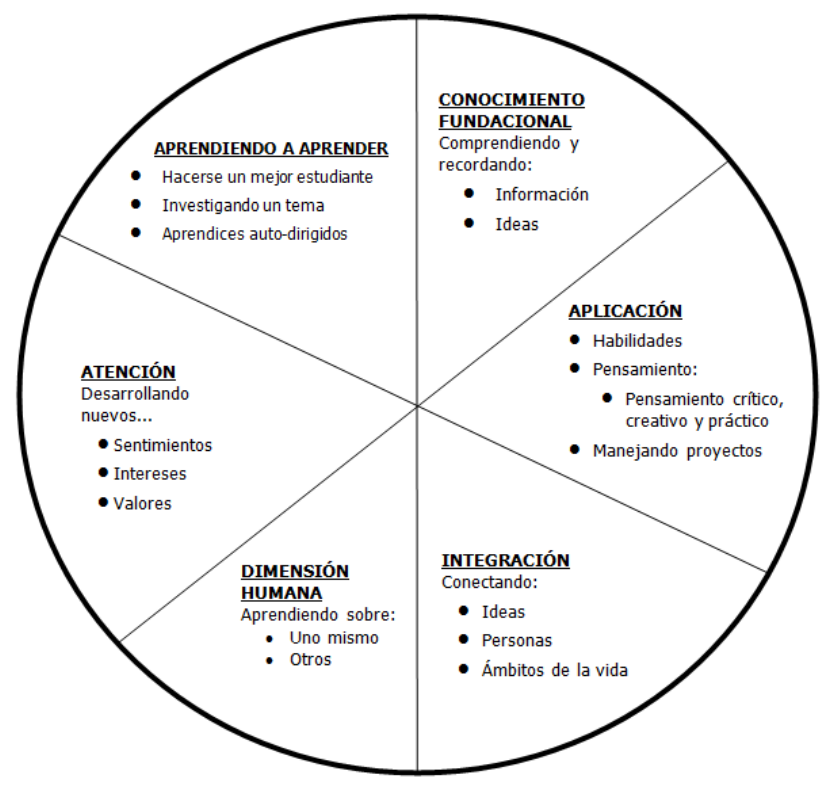

Figura. 3. Taxonomía del aprendizaje significativo.

Nota: Esta taxonomía se adapta al modelo constructivista de enseñanza-aprendizaje de la institución educativa, ya que su misión es la formación integral de profesionistas.

Fuente: Elaboración propia.

Tabla 1. Ejemplos de objetivos específicos por tema para asignaturas del área de la salud

\begin{tabular}{lc}
\hline \multicolumn{1}{c}{ ASIGNATURA } & OBJETIVOS ESPECífICOS (EJEMPLOS) \\
\hline & El estudiante conocerá las funciones y generalidades de la anatomía y \\
& comprenderá los elementos anatómicos de la cabeza y el cuello, los \\
& miembros superiores e inferiores, dorso, y columna vertebral, piel y \\
Anatomía & faneras y los aparatos auditivo y vestibular, visual, olfatorio, bucal y \\
& nasal para desarrollar habilidades de identificación, investigación e \\
& integración de las relaciones anatómicas con la práctica clínica. \\
& El estudiante identificará las técnicas radiográficas utilizadas \\
& comúnmente para la práctica odontológica así como desarrollará la \\
& habilidad necesaria para utilizarlas como medio auxiliar para el \\
Radiología & diagnóstico clínico. \\
& El alumno conocerá y aplicará en la clínica los procedimientos básicos \\
& para la realización de una extracción simple y compleja, así como el \\
Exodoncia II & tratamiento de pacientes con enfermedades sistémicas y el manejo de \\
& urgencias dentro de la clínica. \\
Taller de Análisis y & El estudiante aprenderá la importancia del agua en la composición de \\
Química de los & un alimento para conservar sus características organolépticas y \\
Alimentos I & nutrimentales. \\
\hline
\end{tabular}

Nota: Se observa un mayor empleo de verbos del nivel taxonómico de conocimientos y muy poca aplicación de verbos de otras esferas taxonómicas para un desarrollo integral de los estudiantes. Fuente: Elaboración propia.

Usando el principio de diseño retrospectivo, el siguiente paso en el proceso de toma de decisiones sugerido por Fink es la retroalimentación y la evaluación. La siguiente pregunta básica que se debe formular el docente es ¿Qué deben demostrar los estudiantes para alcanzar las metas de aprendizaje que se definieron para el curso?. Esto puede requerir algunos exámenes escritos con lápiz y papel, pero requiere el empleo de 
otras formas de evaluación, incluyendo actividades de reflexión, reportes de prácticas, ensayos, estudio de casos, etc., para que sea formativa en lugar de sumativa. Esto nos dará claridad de lo que constituye un desempeño exitoso del alumnado y es más fácil establecer posteriormente las actividades de enseñanza aprendizaje. La propuesta de metas de aprendizaje significativas y procedimientos de evaluación efectivos, permite incorporar más fácilmente algunos tipos de aprendizaje activo en la asignatura.

Por ello, la propuesta del formato de planeación didáctica incluyó como siguiente apartado (cuerpo formal), la definición de las principales metas de aprendizaje y las formas de evaluación de los aprendizajes como la visión panorámica de lo que el docente desea lograr al terminar el semestre y que sea recordado 2 a 3 años después de cursar la asignatura, así como la forma global de evaluar dichos aprendizajes (figura 4).

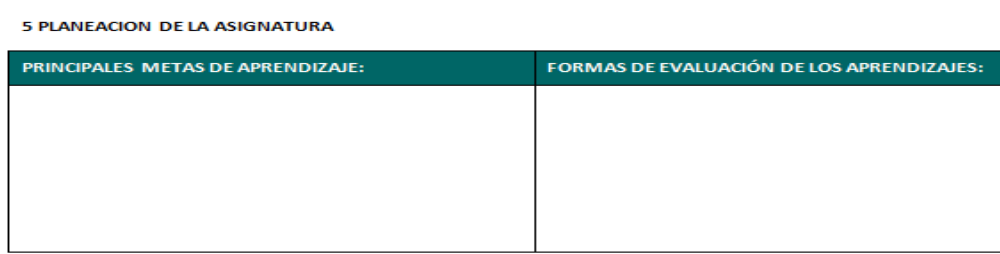

Figura. 4. Planeación de la asignatura

Fuente: Elaboración propia.

Para facilitar la comprensión del formato por parte de los estudiantes, se separaron los siguientes apartados del formato por cada periodo de exámenes parciales, lo que incluye el nombre del tema, los subtemas, los objetivos específicos del tema, el número de sesión, la fecha y los contenidos temáticos por sesión, las actividades de enseñanza-aprendizaje frente a docente e independientes, los recursos y la bibliografía complementaria. Al final de cada periodo parcial, se incluyó un apartado con los productos y criterios para la evaluación formativa del curso, los resultados de aprendizaje y las evidencias de aprendizaje (figura 5).

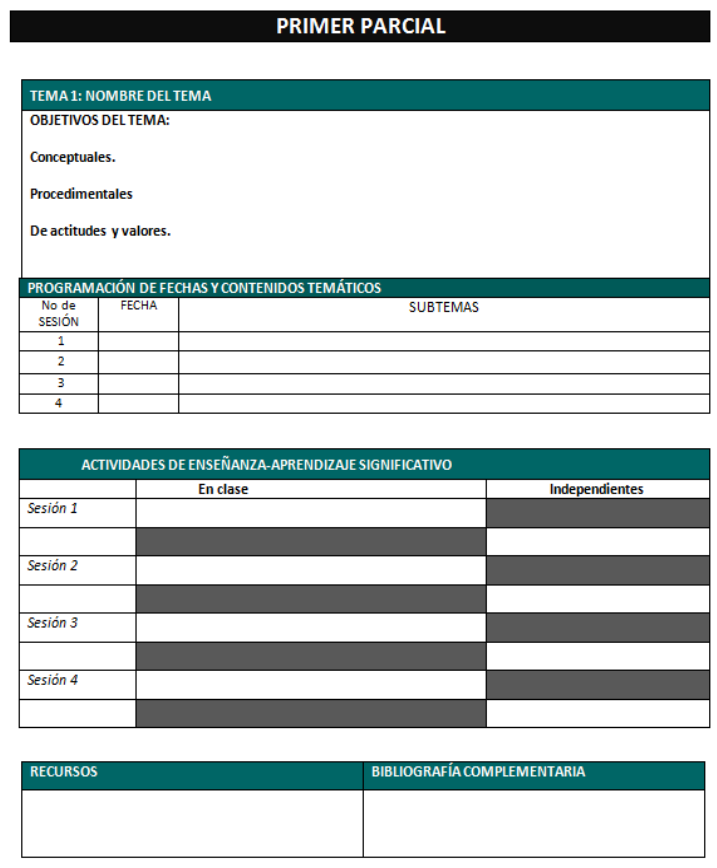

Figura 5. Primer parcial

Fuente: Elaboración propia. 


\subsection{Secciones por tema o período parcial}

\section{Sección de objetivos del tema}

Este apartado es un elemento complejo de llenar para algunos docentes no acostumbrados a la terminología empleada. De acuerdo a Zabalza (2004), su sentido es hacer explícitas tanto los objetivos que se desean que los alumnos alcancen, como las condiciones en las que se desea que lleven a cabo el proceso de aprendizaje. Además de los contenidos que deben dominar, sugiere incluir en los objetivos tres tipos de ganancias: objetivos relacionados con los nuevos conocimientos o habilidades a adquirir en la asignatura, objetivos relacionados con el dominio de ciertas herramientas de aprendizaje y/o de formación y objetivos vinculados a valores o actitudes importantes en función de la asignatura o de su sentido en el Plan de estudios.

Zarzar (2006) define las cinco habilidades mínimas necesarias para que un profesor pueda desarrollar su labor docente, desde la perspectiva del aprendizaje significativo:

- Definir claramente los objetivos de aprendizaje.

- Diseñar el plan de trabajo de un curso escolar y redactar el programa para los alumnos.

- Desarrollar el encuadre en las primeras sesiones.

- Diseñar e instrumentar actividades de aprendizaje y de evaluación de los aprendizajes.

- Integrar y coordinar equipos de trabajo y grupos de aprendizaje.

Por su parte, Murillo, Martínez-Garrido y Hernández-Castilla (2011) en su decálogo para la enseñanza eficaz, añade a estos aspectos el compromiso del docente, el clima en el aula, el propiciar la autoestima y las altas expectativas en los estudiantes, la atención a la diversidad, optimizar el tiempo de aprendizaje, uso adecuado de los recursos didácticos y la retroalimentación continua.

Por ello, de los objetivos o metas que plantee un docente va a depender el resto del curso: la estructuración del contenido, la organización del curso, el diseño de actividades de aprendizaje dentro y fuera del aula, los mecanismos y criterios de evaluación, la bibliografía complementaria y los recursos, entre otros.

Los objetivos de aprendizaje los define Zarzar (2006) como aquellas líneas generales que van a orientar el trabajo del docente. Es más importante la respuesta a la pregunta: ¿qué quiero que los estudiantes aprendan?, es decir las metas del curso o asignatura, que la forma en la que los objetivos se redactan. La forma en que se redacte un objetivo no va a cambiar en nada la forma de trabajo del profesor, por lo que enfatiza el significado de fondo del objeto de aprendizaje, más que en la redacción.

En el formato propuesto a la institución educativa se determinó incluir objetivos conceptuales, procedimentales y de actitudes y valores, para propiciar la formación holística de los estudiantes, según se muestra un ejemplo en la figura 6. Para facilitar la labor de los docentes en la redacción de objetivos específicos de aprendizaje, se sugirió llevar a cabo un catálogo de objetivos de enseñanza aprendizaje, que sirvan como ejemplos de la forma de redactarlos. 


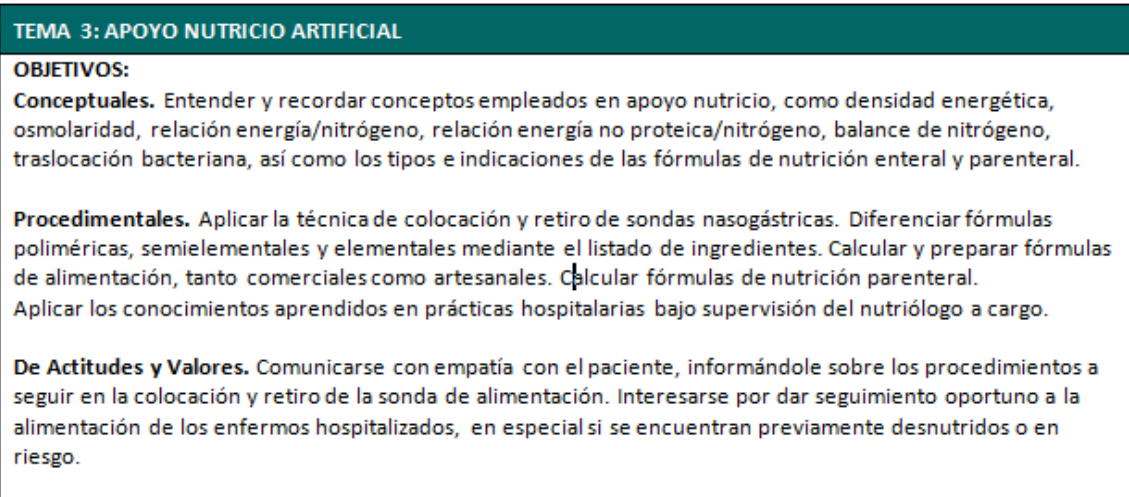

Figura. 6. Ejemplo de objetivos específicos sugeridos en el formato de planeación didáctica

Fuente: Elaboración propia.

\section{Sección de programación de fechas y contenidos temáticos de la asignatura}

En esta sección del formato se debe especificar el listado de temas y subtemas que el docente desea que los estudiantes trabajen bajo su tutela y de forma independiente. Esto debe planearse tomando en cuenta el tiempo disponible para la asignatura y por lo tanto, a los créditos que se cubren del total del plan de estudios. Es necesario orientarse a revisar los contenidos más básicos e importantes para permitir que el estudiante continúe el aprendizaje por su cuenta. El docente debe tener la capacidad de diferenciar los elementos básicos y los complementarios de su disciplina. Zabalza (2004) sugiere diseñar un listado de contenidos por categorías, distinguiendo entre contenidos esenciales, contenidos necesarios y contenidos de ampliación, remarcando la relación entre ellos.

\section{Actividades de enseñanza-aprendizaje significativos}

La metodología a emplear dentro de las actividades de enseñanza-aprendizaje, clarifican y hacen explícita la "coreografía didáctica" del docente; la forma en que se organizan los dispositivos a su alcance para propiciar el aprendizaje de los estudiantes. Zabalza (2004) recomienda utilizar al menos tres formatos metodológicos simples: lección magisterial, trabajo en grupo y el estudio independiente de cada estudiante. Las estrategias deben promover la progresiva autonomía de los estudiantes y deben ir variando de los primeros años a los últimos años de la licenciatura. La motivación e implicación de los estudiantes está muy relacionada con estos diagramas didácticos; las clases demasiado homogéneas resultan muy pesadas y desmotivadoras. Se recomienda introducir debates, lecturas, trabajo en grupo, manejo de fuentes, síntesis y diferenciar las actividades que realizará el docente como lecciones magistrales, apoyos virtuales, de los que se pide que hagan los estudiantes: trabajo en grupo o individual, búsqueda de información, debates, realización de trabajos, visitas, prácticas, etc.

Las planeaciones didácticas analizadas en el campus incluyeron una limitada variedad de actividades de enseñanza aprendizaje y no distinguían de manera clara los tiempos destinados a realizar actividades independientes por parte de los estudiantes. En la práctica, los estudiantes suelen quejarse de una sobrecarga de trabajo independiente, en especial en las semanas de evaluaciones parciales y al final del periodo semestral. Los créditos de una asignatura están definidos por la suma de horas de trabajo frente a 
docente (tanto teóricas como prácticas) y las de trabajo independiente, durante el periodo escolar y el número de semanas académicas. Por ejemplo, si una asignatura se imparte con 7 horas frente a docente ( 4 teóricas y 3 prácticas) y 4 horas de trabajo independiente, el total de horas por semana es de 11. Esto significa que el docente debe planear actividades independientes que no consuman más de 4 horas semanales, ya que los demás profesores que imparten clases en el mismo grupo, también requieren de un tiempo dedicado a sus asignaturas. El análisis de la malla curricular del plan de estudios de las diferentes licenciaturas, permitieron comprobar el efecto de sobrecarga de trabajo para los estudiantes, como se ejemplifica en el tabla 2.

Tabla 2. Análisis de la malla curricular de un semestre de una licenciatura del área de la salud

\begin{tabular}{|c|c|c|c|c|c|c|}
\hline & \multirow[t]{2}{*}{ Clave } & \multirow[t]{2}{*}{ SERIACIÓN } & \multicolumn{2}{|c|}{$\begin{array}{l}\text { ACTIVIDADES BAJO } \\
\text { CONDUCCIÓN DEL } \\
\text { DOCENTE }\end{array}$} & \multirow[t]{2}{*}{$\begin{array}{c}\text { ACT. } \\
\text { INDEP. }\end{array}$} & \multirow[t]{2}{*}{ CRÉDS. } \\
\hline & & & Teóricas & Prácticas & & \\
\hline $\begin{array}{l}\text { Taller de } \\
\text { Tecnología en } \\
\text { Alimentos }\end{array}$ & $\begin{array}{l}\text { TEA } \\
132\end{array}$ & TEA 130 & 3 & 4 & 4 & 11 \\
\hline $\begin{array}{l}\text { Taller de } \\
\text { Fisiopatología y } \\
\text { Dietoterapia I }\end{array}$ & $\begin{array}{c}\text { NIE } \\
119\end{array}$ & NIS1 14 & 4 & 3 & 4 & 11 \\
\hline $\begin{array}{l}\text { Legislación } \\
\text { Alimentaria }\end{array}$ & $\begin{array}{c}\text { SEA } \\
136\end{array}$ & & 2 & 1 & 2 & 5 \\
\hline $\begin{array}{l}\text { Políticas y } \\
\text { Programas de } \\
\text { Nutrición y } \\
\text { Alimentación }\end{array}$ & $\begin{array}{c}\text { AGS } \\
127\end{array}$ & AGS 125 & 3 & 2 & 2 & 7 \\
\hline $\begin{array}{l}\text { Taller de } \\
\text { Educación en } \\
\text { Nutrición }\end{array}$ & $\begin{array}{c}\text { AGS } 12 \\
6\end{array}$ & NIS 114 & 3 & 2 & 2 & 7 \\
\hline $\begin{array}{l}\text { Comunicación } \\
\text { Interpersonal }\end{array}$ & $\begin{array}{c}\mathrm{COM} 2 \\
\mathrm{OO}\end{array}$ & & 3 & $\mathrm{O}$ & 2 & 5 \\
\hline Total & & & 18 & 12 & 16 & 46 \\
\hline
\end{tabular}

Fuente: Elaboración propia.

Podemos observar que en este semestre, los estudiantes deben dedicar 30 horas de actividades frente a docente y 16 horas independientes, lo que asciende a 46 horas semanales, que superan una jornada laboral de 40 horas por semana. Si a esto se le suma que los docentes solicitan trabajos o actividades independientes que impliquen más tiempo del descrito en la malla curricular, los estudiantes tendrán realmente una sobrecarga de actividades y empezarán a fallar en la entrega de trabajos o reportes.

Fink sugiere que en la planeación se dividan las actividades frente a docente y las independientes en un formato tipo "greca en s" que defina los tiempos estimados para cada actividad, como se muestra en la siguiente figura (figura 7). Sin embargo, el formato de greca en forma de "Tope de Castillo" no permite realizar secuencias largas de más de 3 o 4 sesiones, por lo que en la nueva propuesta de planeación didáctica se sugirió utilizar el formato en forma vertical, empleando el mismo esquema de horas frente a docente e independientes separadas y empleando espacios oscuros que facilitaran la comprensión del llenado del formato.

Esto permitió al docente limitar las actividades independientes al número de horas asignadas por semana, como se muestra en el siguiente ejemplo (tabla 3). 
Figure - The "Castle Top" Template for Creating an Instructional Strategy

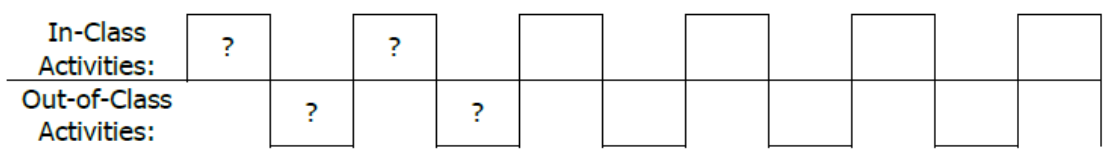

Action: Sketch out a sequence of varied activities using some of the learning activities you identified in Phase I.

Figura 7. Planeación de actividades frente a docente e independientes, de acuerdo al modelo de Fink

Nota: El modelo de tope de castillo o greca en S permite diferenciar las actividades frente a docente e independientes, de modo que se detecten sobrecargas de trabajo independiente que afecten el desempeño escolar del grupo.

Fuente: Elaboración propia.

Tabla 3. Actividades de enseñanza-aprendizaje significativo. Ejemplo de llenado de formato con actividades frente a docente e independientes equilibradas

\begin{tabular}{|c|c|c|c|}
\hline & & EN CLASE & INDEPENDIENTES \\
\hline \multirow[t]{2}{*}{$\overline{\tilde{0}}$} & $2.5 h$ & $\begin{array}{l}\text { Encuadre del curso. Dar a conocer } \\
\text { muro de Facebook para la clase } \\
\text { Examen diagnóstico } \\
\text { Expectativas del docente y estudiante } \\
\text { Solicitar al estudiante buscar ejemplos } \\
\text { de dietas para enfermos en libros de } \\
\text { nutrición clínica e internet }\end{array}$ & \\
\hline & $2 h$ & & $\begin{array}{l}\text { Ejemplos de dietas para enfermos. } \\
\text { Búsqueda en libros de nutrición } \\
\text { clínica. Subir tarea al muro de } \\
\text { facebook }\end{array}$ \\
\hline \multirow[t]{2}{*}{ 䜦 } & $2 h$ & $\begin{array}{l}\text { Lluvia de ideas: ejemplos de dietas para } \\
\text { enfermos } \\
\text { Exposición de cátedra: Análisis de las } \\
\text { dietas para enfermos y clasificación } \\
\text { en modificaciones de tipo cualitativo } \\
\text { o cuantitativo. } \\
\text { Foro grupal: Experiencias personales o } \\
\text { familiares con dietas hospitalarias. }\end{array}$ & \\
\hline & $2 h$ & & $\begin{array}{l}\text { Mapa mental de dietas hospitalarias y } \\
\text { problemas por los cuales los } \\
\text { pacientes se desnutren }\end{array}$ \\
\hline$\stackrel{\substack{0 \\
\infty}}{\infty}$ & $1 h$ & $\begin{array}{l}\text { Trabajo en grupo: Conclusiones sobre } \\
\text { el mapa mental. Subir conclusiones al } \\
\text { muro de facebook. } \\
\text { Inicio de próximo tema. }\end{array}$ & \\
\hline
\end{tabular}

Fuente: Elaboración propia.

De acuerdo al momento de uso y presentación en la sesión de clase, las estrategias de enseñanza pueden incluirse antes, durante y después de un contenido curricular específico (Díaz Barriga, 1998), como se muestra en la figura 8. Se recomendó usar entre dos y cuatro tipos de estrategias de enseñanza en una sesión, para lograr una clase dinámica y motivante. En ellas, es necesario equilibrar actividades individuales y grupales o en equipos. Las estrategias seleccionadas también deben contemplar el tiempo y los recursos disponibles para la sesión o clase. Por ello, se debe contemplar el tiempo 
destinado a la estrategia preinstruccional (aproximadamente $10 \%$ del tiempo de la sesión), las coinstruccionales (70 a $80 \%$ ) y las post-instruccionales (10 a $20 \%$ ).

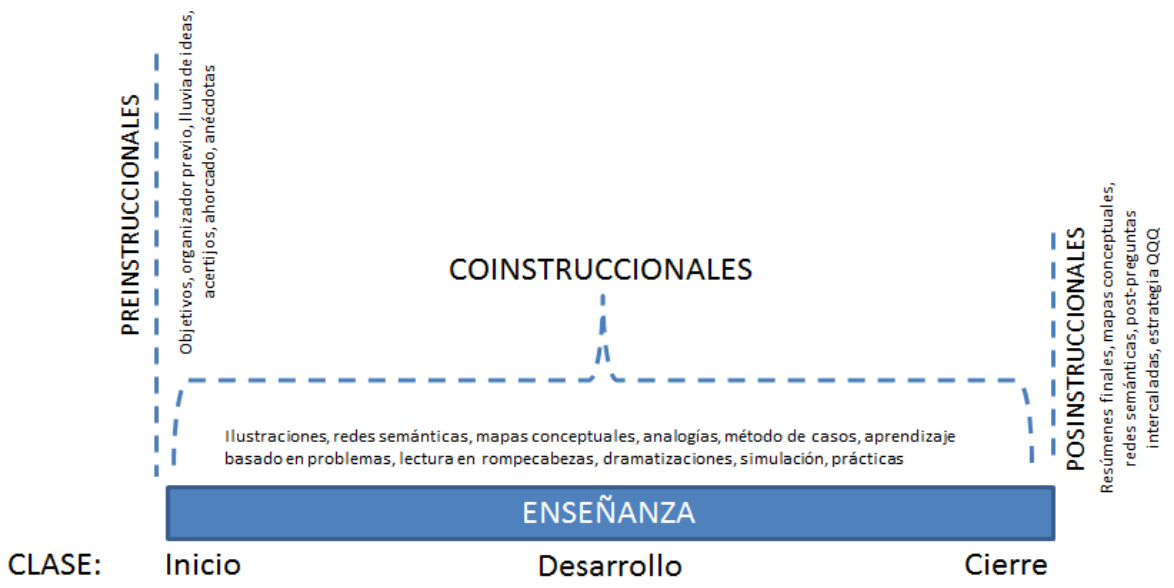

Figura 8. Clasificación de estrategias de acuerdo al momento específico de la clase o

sesión

Fuente: Elaboración propia.

En la modalidad presencial o clases frente a docente, las actividades se clasifican de acuerdo al momento específico en pre-instruccionales, que se realizan por breves periodos al inicio de la sesión, las co-instruccionales, que ocupan la mayor parte del desarrollo de la clase y las actividades de cierre o post-instruccionales. El manejo de los tiempos en la sesión ayuda al docente a planear mayor variedad de actividades y a controlar el tiempo determinado para cada una, mejorando la secuencia y estructura de la clase.

En el formato de llenado, se sugirió incluir las actividades de inicio, desarrollo y cierre de la sesión, de manera que el formato sustituya de manera parcial el empleo del plan de clase, junto con una descripción detallada de recursos por tema. Como parte de los apoyos ofrecidos a los docentes, se realizaron catálogos de estrategias de enseñanza aprendizaje de acuerdo al momento específico de la sesión.

De acuerdo a la guía para diseñar cursos para el aprendizaje significativo de Fink (2006), las actividades de enseñanza aprendizaje deben ir más lejos que solo lecturas y discusiones. El aprendizaje activo es una forma más potente de lograr aprender y retener por periodos mayores, si adquieren un rol activo en vez de pasivo e implica involucrar a los estudiantes en hacer y pensar acerca de las cosas que están haciendo. Hacer cosas, se refiere a llevar a cabo actividades como debates, simulaciones, diseños guiados, solución de problemas en grupos pequeños, estudios de casos. Cuando los estudiantes escuchan una lectura o leen un texto, están recibiendo información e ideas, lo cual es una parte importante del proceso, pero en esencia es relativamente pasivo. Si añadimos algún tipo de aprendizaje experiencial y oportunidades para el diálogo reflexivo, el aprendizaje se vuelve más activo (figura 9).

Entre las actividades que aportan información e ideas, se encuentran fuentes primarias y secundarias que hay que acceder en clase, fuera de clase o en línea. En cuanto a experiencias, se recomienda introducir actividades de hacer, observar, situaciones actuales o simuladas, experiencias ricas en aprendizajes. Respecto al diálogo reflexivo, 
emplear escritos breves, portafolios de aprendizaje, reportajes, reflexión sobre la asignatura o el proceso de aprendizaje. El diálogo reflexivo sobre sí mismo, puede incluir cuestionamientos como ¿qué estoy aprendiendo?, ¿qué valor tiene este conocimiento?, ¿Cómo aprendo mejor y más cómodamente?, ¿qué más necesito hacer para aprender?.

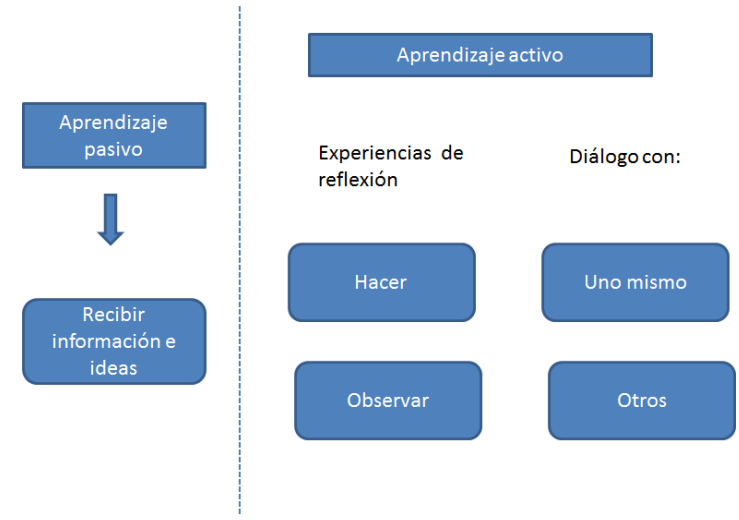

Figura 9. Actividades de aprendizaje pasivas y activas

Nota: El tipo de actividades incluidas en el aula pueden propiciar aprendizajes significativos, si favorecen experiencias de mayor participación y reflexión por parte de los estudiantes, tanto en forma individual, como colaborativa.

Fuente: Elaboración propia.

A continuación se presentan algunas actividades de aprendizaje sugeridas para el aprendizaje activo e integral empleadas en la capacitación docente (tabla 4):

Tabla 4. Actividades de aprendizaje sugeridas para un aprendizaje activo e integral

\begin{tabular}{|c|c|c|c|c|c|}
\hline & \multirow{2}{*}{$\begin{array}{c}\text { OBTENER } \\
\text { INFORMACIÓN O } \\
\text { IDEAS }\end{array}$} & \multicolumn{2}{|c|}{ EXPERIENCIA } & \multicolumn{2}{|c|}{ DIALOGO DE REFLEXIÓN } \\
\hline & & Hacer & Observar & Si mismo & Otros \\
\hline Directo & $\begin{array}{l}\text { Fuentes primarias } \\
\text { Fuentes secundarias }\end{array}$ & $\begin{array}{l}\text { Escenarios } \\
\text { reales }\end{array}$ & Fenómenos & \multirow[b]{2}{*}{$\begin{array}{l}\text { Pensamiento } \\
\text { reflexivo } \\
\text { reportajes }\end{array}$} & \multirow[b]{2}{*}{$\begin{array}{l}\text { Diálogo } \\
\text { (dentro o } \\
\text { fuera de } \\
\text { clase) }\end{array}$} \\
\hline $\begin{array}{c}\text { Indirecto/ } \\
\text { Varios }\end{array}$ & $\begin{array}{l}\text { Datos y fuentes } \\
\text { secundarios } \\
\text { Lecturas, libros de } \\
\text { texto }\end{array}$ & $\begin{array}{l}\text { Estudio de } \\
\text { casos } \\
\text { Simulaciones } \\
\text { Juego de } \\
\text { papeles }\end{array}$ & $\begin{array}{l}\text { Historias } \\
\text { (mediante } \\
\text { películas, } \\
\text { historias } \\
\text { contadas o } \\
\text { literatura) }\end{array}$ & & \\
\hline En línea & $\begin{array}{l}\text { Cursos en línea } \\
\text { Internet }\end{array}$ & $\begin{array}{l}\text { El profesor pu } \\
\text { estudiantes } \\
\text { experiencia } \\
\text { Los estudiant } \\
\text { involucrarse } \\
\text { experiencias } \\
\text { través de la }\end{array}$ & $\begin{array}{l}\text { de asignar a } \\
\text { una } \\
\text { recta } \\
\text { pueden } \\
\text { on } \\
\text { directas a } \\
\text { d }\end{array}$ & \multicolumn{2}{|c|}{$\begin{array}{l}\text { Los estudiantes pueden } \\
\text { reflejarse e involucrarse } \\
\text { con diferentes tipos de } \\
\text { diálogo en línea }\end{array}$} \\
\hline
\end{tabular}

Fuente: Elaboración propia.

Para incrementar las experiencias ricas en aprendizajes Fink (2006) recomienda utilizar debates, juego de papeles, simulaciones y dramatizaciones en el aula, mientras que sugiere el aprendizaje en el servicio, observaciones situacionales y proyectos auténticos como actividades independientes. 


\section{Recursos}

La revisión de los recursos empleados por los docentes de las diferentes licenciaturas permitió evidenciar que por lo general emplean siempre las mismas en la mayoría de las sesiones de clase. Esto puede deberse a que la una gran parte de los docentes desconoce todos los recursos disponibles en el campus y en la institución para poder diversificar las actividades programadas, por lo que se sugirió integrar un catálogo de recursos disponibles, así como el departamento o área responsable de su control, para que se puedan solicitar y emplear como recursos en clase.

\section{Bibliografía básica y complementaria}

La bibliografía básica se consideró como aquella que está registrada en el anexo respectivo del Plan de estudios de cada licenciatura. Este apartado fue trasladado a la plantilla estandarizada del formato. El resto de las referencias sugeridas por el profesor, que incluyen libros, artículos, videos, enlaces a páginas de internet, entre otros, se clasificaron como bibliografía complementaria y se especificaron al final de cada tema o unidad (figura 10).

\begin{tabular}{|l|l|}
\hline RECURSOS & BIBLIOGRAFÍA COMPLEMENTARIA \\
\hline Caso clínico & Tapia Jurado J. Manual de Maniobras médico- \\
$\begin{array}{l}\text { Aula con acceso a internet o centro de cómputo } \\
\text { Catálogo de fórmulas de alimentación de Abbott } \\
\text { Sonda de alimentación }\end{array}$ & quirúrgicas. Capítulo 3. Colocación de sonda \\
Estetoscopios, tela adhesiva, guantes & nasogástrica. Editorial Alfil. Recuperado de: \\
desechables & MANIOBRAS-MEDICO-QUIRURGICAS/ \\
Bolsas de infusión, fórmula enteral comercial & Díaz Reynoso R (2013), Endonutrición. A poyo \\
Alimentos & nutricio. Ed. Manual Moderno. \\
Video sobre preparación de fórmulas & Canul Medina G (2009). Glutamina en nutrición \\
parenterales & clínica. Rev. Endocrinología y Nutrición \\
Bolsa de nutrición parenteral prediseñada & 17(4):161-9. \\
\hline
\end{tabular}

Figura 10. Ejemplo de recursos y referencias bibliográficas en la planeación didáctica propuesta

Fuente: Elaboración propia.

\subsection{Productos y criterios para la evaluación formativa del curso por periodo parcial}

Al finalizar un periodo parcial de actividades, se incluyó el siguiente apartado que contiene la información integrada respecto a la evaluación formativa de los aprendizajes. Este segmento incluyó los porcentajes destinados a las diferentes actividades realizadas por los estudiantes, bajo su conducción y de manera independiente, de modo que la suma de todos los factores den como resultado la evaluación formativa del periodo parcial. Así mismo, se indicaron los resultados del aprendizaje y las evidencias de aprendizaje.

En este apartado se incluyó una tabla que especifica claramente los aspectos a evaluar, la forma de hacerlo, los criterios a utilizar y el peso de cada aspecto evaluado.

Las actividades de enseñanza con docente se retomaron de la información descrita en el programa académico, ajustando las cifras a las actividades reales programadas por el docente, mientras que las actividades independientes se establecieron directamente a partir de las actividades programadas por el profesor. La suma de factores corresponde al $100 \%$ (figura 11 ). 


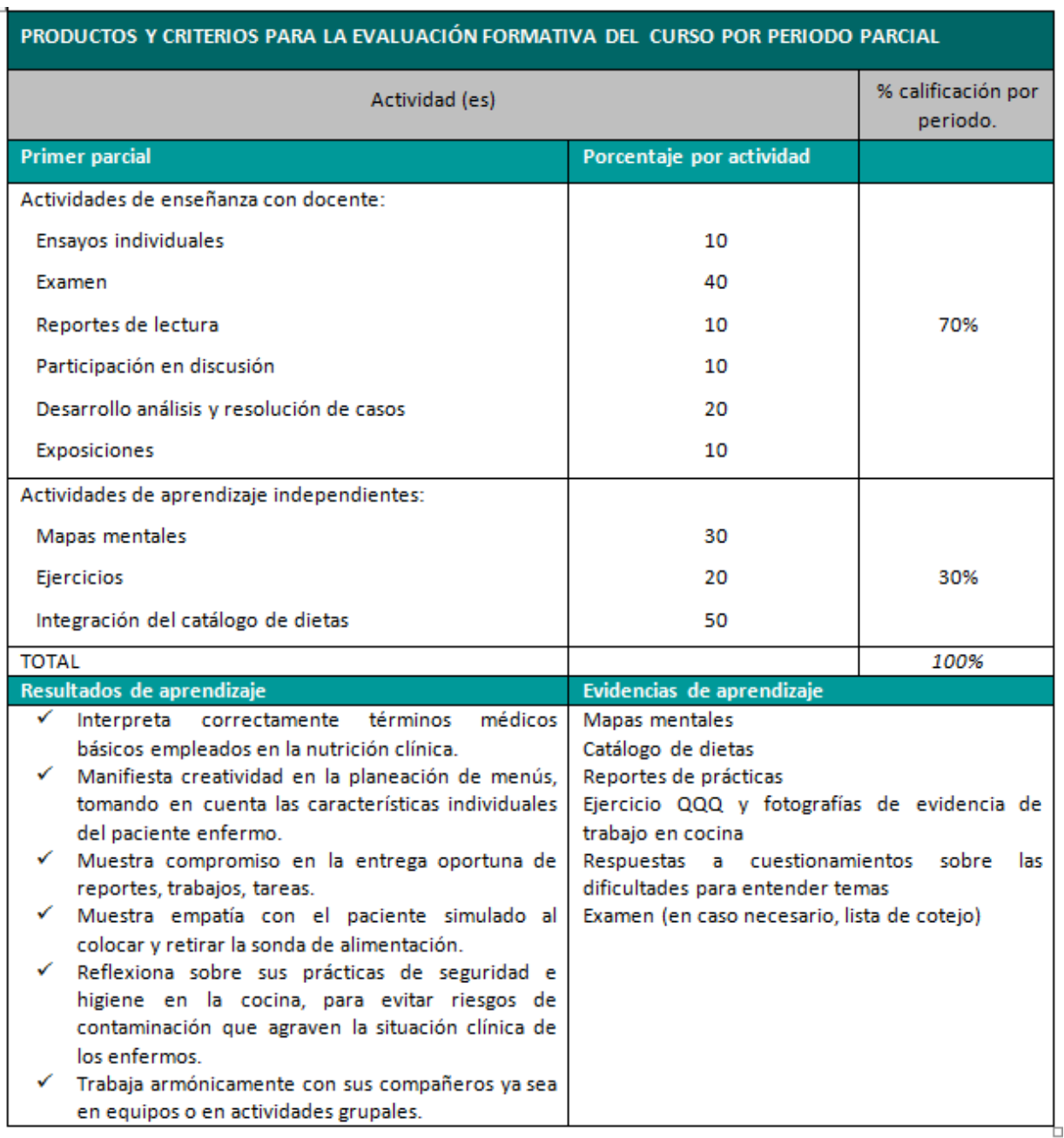

Figura 11. Ejemplo de llenado de los productos y criterios para la evaluación formativa de un curso por periodo parcial

Fuente: Elaboración propia.

\section{Resultados de aprendizaje}

Los resultados de aprendizaje son enunciados acerca de lo que se espera que el estudiante sea capaz de hacer, comprender y/o sea capaz de demostrar una vez terminado un proceso de aprendizaje. Cada resultado de aprendizaje comienza con un verbo de acción, seguido por el complemento del verbo y por una frase que le provea contexto.

Los resultados de aprendizaje pueden ser específicos o genéricos (Andrade, 2013). En el primer caso, implica la investigación como eje transversal para el desarrollo de cada una de las asignaturas, esto apunta a un nuevo perfil profesional, que deben saber cómo resolver los problemas que se enfrenta diariamente en el ámbito de su profesión.

En los formatos de planeación didáctica analizados, se encontró confusión por parte de los docentes respecto a la información que debieron llenar en los resultados de aprendizaje, ya que algunos los redactaron como evidencias y otros en forma de logros obtenidos por los estudiantes, lo que indica la importancia de un instructivo de llenado o curso de capacitación previo. 


\section{Evidencias del proceso de enseñanza aprendizaje}

De acuerdo a Fink (2006), las asignaturas o cursos basados en el aprendizaje requieren una aproximación más especializada respecto a la forma de evaluar y retroalimentar a los estudiantes, que los exámenes parciales aplicados en un curso tradicional. A este sistema se le conoce como evaluación educativa y se caracteriza por buscar la visión prospectiva; es decir, el tipo de situaciones en las que se espera que el estudiante deba saber o aplicar ese conocimiento. Una vez determinado, se crea un cuestionamiento o problema que replica situaciones de la vida real, tan cercanamente como sea posible. Pueden ser más o menos estructurados según sea el caso. Además, debe incorporar ejercicios, preguntas y/o problemas que crean un contexto de la vida real para un determinado aspecto, problema o decisión que deberán ser tomadas.

Así mismo, Fink (2006) recomienda involucrar a los estudiantes en su autoevaluación, aprendiendo a ser críticos de su autodesempeño y a crear estándares de calidad en su propia área de trabajo. La evaluación requiere de retroalimentación al estudiante, que debe ser de alta calidad incluyendo que sea frecuente (diaria, semanal, quincenal), inmediata (tan pronto como sea posible), discriminante (que determine la diferencia entre lo bien hecho y lo que no se hizo correctamente) y con cariño o empatía respecto a la forma en la que retroalimentamos a los estudiantes.

Las evidencias de aprendizaje incluyen todos aquellos documentos (exámenes escritos, reportes de prácticas, ensayos), imágenes (videos, fotografías), registros digitales (muros, blogs), etc., las cuales se recopilan a lo largo del periodo académico como evidencias objetivas de la labor docente.

\subsection{Evaluación sumativa}

Finalmente se integró al formato sugerido, la forma en las que los diferentes componentes evaluados en el curso o asignatura se convierten en una calificación final, de acuerdo a los criterios establecidos en el reglamento de la institución educativa. Debido a que es un componente no modificable de la planeación didáctica, se esquematiza en color gris, lo que significa que es parte de las secciones que debe llenar el coordinador académico. En la tabla 5 se ejemplifica la evaluación sumativa incluida en el formato.

Tabla 5. Evaluación sumativa

\begin{tabular}{lc}
\hline \multicolumn{1}{c}{ CALIFICACIÓN FINAL } & PROCENTAJE POR EXAMEN \\
\hline Primer examen parcial & $20 \%$ \\
Segundo examen parcial & $20 \%$ \\
Tercer examen parcial & $20 \%$ \\
Examen final & $40 \%$ \\
Total & $100 \%$ \\
\hline Fuente: Elaboración propia.
\end{tabular}

Fuente: Elaboración propia.

\section{Discusión y conclusiones}

La formación de profesionistas exitosos y con alto nivel de capacitación y compromiso, es el resultado de la consolidación de una serie de intereses, expectativas, valores y demandas de parte de las organizaciones internacionales, la sociedad, los padres de familia, los futuros empleadores, el sistema educativo nacional, la institución de educación, los profesores y otros expertos. La institución educativa es la responsable de 
dicha integración, a través del esfuerzo coordinado de sus recursos, su plantilla docente, su filosofía institucional y su modelo educativo.

A la institución educativa le compete engarzar los elementos que conforman su plan de estudios, desde el nivel del currículum, hasta al nivel del programa de una asignatura. Las cartas descriptivas o planeaciones didácticas, son el instrumento adecuado para explicitar intencionalidades, objetivos, métodos y criterios de una asignatura, en un marco educativo específico, por lo que es un documento interno de mucho valor para los actores del proceso de enseñanza aprendizaje, así como para la gestión educativa a nivel superior.

La planeación didáctica juega por lo tanto, un importante papel en el desarrollo de una docencia de calidad. Debe ayudar a transformar la docencia de modo que el eje fundamental no sea la enseñanza, sino el aprendizaje. Los estudiantes deben ser preparados para un tipo de aprendizaje autónomo, pero acompañado y guiado por los docentes; dando igual o más importancia al dominio de la forma en la que aprenden, que a la mera acumulación de contenidos; equilibrar las exigencias de la asignatura y la organización del curso con las condiciones y apoyos que se ofrecen y favorecer la progresividad y la continuidad en la formación, construyendo la planeación didáctica en función de su aportación al perfil profesional de la carrera.

A nivel de la educación superior, la capacitación pedagógica y didáctica del docente es de suma importancia, dado que la gran mayoría son profesionales con experiencia en su campo laboral, pero sin preparación en la impartición de clases, en el llenado de documentos como la planeación didáctica, en estrategias de enseñanza-aprendizaje activas que desarrollen aprendizajes significativos en los estudiantes y que propicien la formación integral del egresado. La mayoría de los docentes universitarios fueron formados bajo esquemas de enseñanza tradicional, por lo que no cuentan con experiencia en modelos de enseñanza con enfoques constructivistas y replican en sus clases, la forma en que ellos mismos fueron enseñados.

Entender a la planeación didáctica como una herramienta de gran utilidad, al comprender todos los elementos que la contienen y convertirse en una guía indispensable en la labor docente, debe uno de los objetivos primordiales de capacitación para las instituciones educativas a nivel superior. El análisis de las planeaciones didácticas y su comparación con los elementos de los syllabi, permitió detectar algunas debilidades de formato y de llenado, que fueron la base para rediseñar la estructura, el orden de los contenidos, fusionar nuevos elementos aportados por los syllabi y el plan de clase, que le den más sentido al docente y mayor congruencia y significado al estudiante al momento de leerlo y utilizarlo.

Para su correcto uso, el formato se complementó con catálogos que incluyeron recursos disponibles en el campus, ejemplos de estrategias, objetivos y resultados de aprendizaje, así como de un instructivo detallado de su llenado. Queda como siguiente etapa comprobar la comprensión y el apego hacia el instrumento por parte del profesorado, así como detectar futuras oportunidades de reajustarlo, en la medida en que los profesores adquieran mayor experiencia en su llenado y lo utilicen como una verdadera guía de su práctica docente. 


\section{Referencias}

Andrade, X. (2013). Guía para el desarrollo de actividades de aprendizaje. Recuperado de: http://www.uce.edu.ec/documents/22994/5f96b84b-5224-4acb-bfc6-9fa79523738c

Casanova, M. A. (2012). El diseño curricular como factor de calidad educativa. REICE. Revista Iberoamericana sobre Calidad, Eficacia y Cambio en la Educación, 1O(4), 7-20.

Díaz-Barriga, F. (1998). Estrategias docentes para un aprendizaje significativo. Una interpretación constructivista. Ciudad de México: McGraw Hill.

Díaz-Barriga, F. y Rigo M. A. (2011). Realidades y paradigmas de la función docente: implicaciones sobre la evaluación magisterial en educación superior. Revista de Educación Superior, 32(3), 53-61.

Fink, L. D. (2006). Fink's 5 principles of fine teaching. Recuperado de: http://tlccvc.org/finksfive.htm

Fink, L. D. (2013). A self-directed guide to designing courses for significant learning. San Francisco, CA: Jossey-Bass.

Gregorutti, G. J. y Bon-Pereira, M. V. (2013). Acreditación de la universidad privada. ¿Es un sinónimo de calidad? REICE. Revista Iberoamericana sobre Calidad, Eficacia y Cambio en Educación, 11(1), 122-139.

Haro del Real, F. J. (1994). Cartas descriptivas ¿Para qué?. Sinéctica, 4, 1-7

Murillo, F. J., Martínez-Garrido, C. y Hernández-Castilla, R. (2011). Decálogo para una enseñanza eficaz. REICE. Revista Iberoamericana sobre Calidad, Eficacia y Cambio en Educación, 9(1), 6-27.

SEP. (2009). Guía para la instrumentación didáctica de los programas de estudio para la formación y desarrollo de competencias profesionales. Recuperado de: http://www.itesca.edu.mx/

SEP. (2013). Planeación educativa. Recuperado de: http://www.dgespe.sep.gob.mx/

UNESCO. (2005). La conceptualización de la UNESCO sobre calidad. Un marco para el entendimiento, el monitoreo, y la mejora de la calidad educativa. París: UNESCO.

Zabalza, M. A. (2004). Guía para la planificación didáctica de la docencia universitaria en el marco del EEES. Ciudad de México: Secretaría de Educación Pública.

Zarzar, C. (2006). La definición de objetivos de aprendizaje. Una habilidad básica para la docencia. Ciudad de México: Universidad Nacional Autónoma de México.

\section{Breve CV de la autora}

\section{Claudia Ascencio Peralta}

Licenciada en Dietética y Nutrición, Escuela de Dietética y Nutrición del ISSSTE. Maestra en Ciencia y Tecnología de Alimentos, Universidad Iberoamericana, Plantel Santa Fe. Coordinadora y profesora de la Escuela de Nutrición de la Universidad Latinoamericana, Campus Cuernavaca. Egresada de la Maestría en Ciencias de la Educación, Universidad Latinoamericana, Campus Norte. Email: claudia.ascencio@ula.edu.mx 